\title{
PET myocardial perfusion quantification: anatomy of a spreading functional technique
}

\author{
L. E. Juarez-Orozco ${ }^{1,2}$ (D - J. R. Cruz-Mendoza ${ }^{3}$ - G. Y. Guinto-Nishimura ${ }^{3} \cdot$ L. Walls-Laguarda ${ }^{3}$. \\ L. J. Casares-Echeverría ${ }^{3}$. A. Meave-Gonzalez ${ }^{4} \cdot$ J. Knuuti $^{1} \cdot$ E. Alexanderson ${ }^{3}$
}

Received: 6 December 2017 / Accepted: 8 January 2018 / Published online: 29 January 2018

(c) The Author(s) 2018. This article is an open access publication

\begin{abstract}
Purpose To summarize the physical principles, imaging method, available tools for and the clinical value of quantitative perfusion evaluation with cardiac PET as well as future aims in the field in a narrative review.

Results Cardiac positron-emission tomography (PET) currently constitutes the reference standard for non-invasive quantitative evaluation of myocardial blood flow. This added modality provides useful information beyond standard semi-quantitative myocardial perfusion evaluation. A description of how the different phases of PET studies are interpreted is provided, as well as a short depiction of the most commonly used radiotracers and the main characteristics affecting their clinical utility. The diagnostic and prognostic utility concerning myocardial perfusion quantified in absolute terms is discussed and the additional contribution of the increasingly spread hybrid equipment is summarized.

Conclusion PET myocardial perfusion represents an excellent noninvasive technique for the evaluation of known or suspected ischemic heart disease, and its clinical application should widen in the near future. The clinical value of PET quantitative perfusion is expected to improve patient outcomes and optimize therapeutic decisions, which constitute key elements for the future of cardiovascular medicine.
\end{abstract}

Keywords Positron-emission tomography $\cdot$ Coronary artery disease $\cdot$ Myocardial perfusion imaging $\cdot$ Myocardial blood flow

\section{Introduction}

Positron-emission tomography myocardial perfusion imaging (PET MPI) has loomed and crystallized within nuclear medicine techniques during the last three decades due to its ability to assess myocardial blood flow (MBF) in absolute terms. Currently, PET MPI represents the gold standard modality for this purpose. By fueling the enthusiasm

\section{E. Juarez-Orozco}

L.E.Juarez.Orozco@gmail.com

1 Turku PET Centre, Turku University Hospital, Kiinamyllynkatu 4-8, 20520 Turku, Finland

2 Department of Nuclear Medicine and Molecular Imaging, University Medical Center Groningen, Groningen, The Netherlands

3 Department of Nuclear Cardiology, National Cardiology Institute "Ignacio Chávez", Mexico City, Mexico

4 Magnetic Resonance Unit, National Cardiology Institute "Ignacio Chávez", Mexico City, Mexico of cardiovascular scientists and physicians, this approach in cardiac imaging has propelled the non-invasive assessment of cardiac physiology and pathophysiology to a new state-of-the-art.

Translational research for MBF quantification has been an intense research topic. The results of such efforts have led to its complete integration into daily practice in a growing amount of selected centers around the world (e.g., Finland, The Netherlands and Sweden) as the intrinsic diagnostic and prognostic added value of the modality has been robustly demonstrated. The present review aims to summarize the evidence behind the increasingly suggested clinical importance of cardiac PET quantitative perfusion in the setting of diagnosis and prognosis of coronary artery disease (CAD). Additionally, the technique's principles, methods and particularities, and future perspectives will be presented. 


\section{PET technology in myocardial perfusion imaging}

PET technology uses coincidence detection of pairs of high-energy photons that are emitted ( $\gamma$-rays with an angle of $180^{\circ}$ between them and energy of $511 \mathrm{keV}$ ) from the "annihilation" of an emitted positron with a free electron as shown in Fig. 1. These positrons arise from the decay of radiopharmaceuticals administered to the patient in order to track (patho)physiological processes.

The two photons are detected in opposite sides of the ring detectors embedded in the camera. Improved detectors (LSO, LYSO or LGSO) are coupled to optimized electronics yield high peak count rates and currently, events can be detected in a 3D mode through the entire cardiac field-of-view and the hybrid composition of the scanners allows for the use of CT to create an attenuation map or transmission image for automatic attenuation losses and scatter correction [1].

\section{Acquisition and reconstruction}

Presently, "list-mode" acquisition is performed to reconstruct static, dynamic and gated images with a single radiotracer injection. In such a modality, all of the acquisition measured coincidences and the time tags are saved in a file for retrospective processing.
Static data are traditionally reconstructed from the final frame $(\approx 900 \mathrm{~ms})$ obtained for visual and semi-quantitative interpretation (\% uptake relative to a reference region, usually the one with the highest uptake). Further, myocardial perfusion absolute quantification is derived from the sequential dynamic first-pass images which, when paired to the EKG or respiratory gating, further permit evaluation of $\mathrm{LV}$ contractile function and even phase synchrony. 3D-mode acquisitions convey higher sensitivity and optimized corrections. In turn, this facilitates reduction in injected doses and therefore optimization of dosimetry (see Dosimetry).

Methods for optimizing image reconstruction have been implemented including filtered-back projection and currently, the widely used iterative reconstruction approaches. Iterative reconstructions deliver images with reduced noise and enhanced myocardium contrast and resolution, with added improved temporal resolution. As such, due to sensitivity to counts and the nature of the detection, PET scanning has proven superior image quality and therefore increased diagnostic accuracy over SPECT [2].

\section{PET imaging protocols}

PET MPI is generally conducted in a two-phase acquisition, i.e., during rest and during stress (either physical or pharmacological). However, it is also possible to conduct a threephase scan which incorporates cold pressor testing (CPT) through sympathetic stimulation. The total acquisition time will, therefore, depend on the number of performed phases,
Fig. 1 PET technology principle. Diagram depicting principles of annihilation and coincidence detection

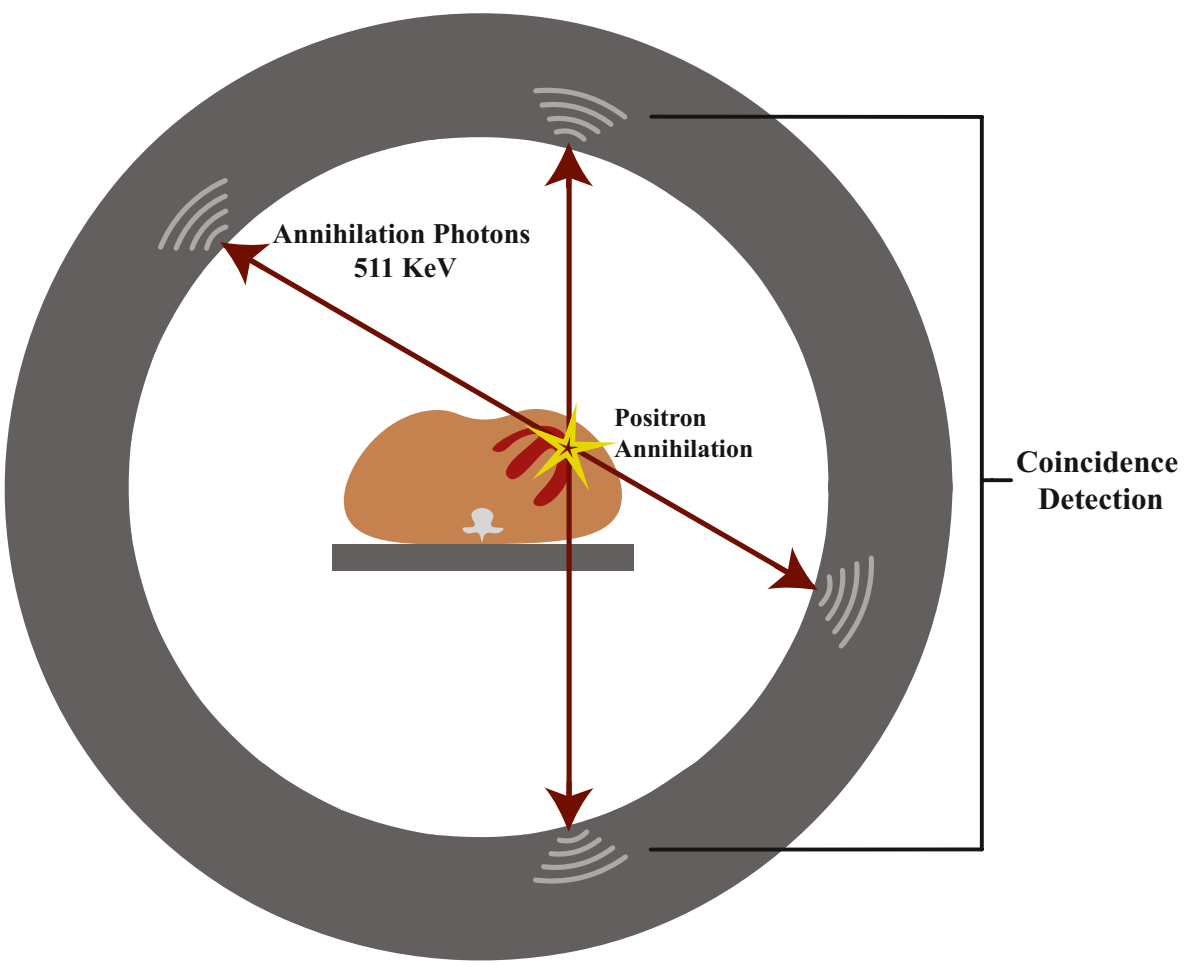


the stress-induction protocol, the length of the dynamic imaging (which depends upon the tracer) and the CT imaging (in hybrid equipment). In average, a full rest-stress and CT acquisition can be performed in 30-40 min [3], a shorter time when compared to SPECT due to the half-life of the radiotracers.

Quantitative perfusion begins with a topogram acquisition for patient positioning. Then, a CT transmission scan is acquired. Afterwards, the radiotracer is injected (25-50 mCi of ${ }^{82} \mathrm{Rb}, 10-30 \mathrm{mCi}$ of ${ }^{15} \mathrm{O}-\mathrm{H}_{2} \mathrm{O}$ or $10-20 \mathrm{mCi}$ of ${ }^{13} \mathrm{~N}$-ammonia) and the rest imaging acquisition is initiated simultaneously (5-6 min for ${ }^{82} \mathrm{Rb}$ or ${ }^{15} \mathrm{O}-\mathrm{H}_{2} \mathrm{O}$ and $10 \mathrm{~min}$ for ${ }^{13} \mathrm{~N}$-ammonia) [4]. Then, stress is induced and a second dose of radiotracer is injected and the stress imaging acquisition is performed. The most common pharmacologic stressor is adenosine administered as a 4- to 6-min infusion of $140 \mu \mathrm{g} /$ $\mathrm{Kg} / \mathrm{min} 2$ or $3 \mathrm{~min}$ before the injection of the radiotracer. Regadenoson constitutes another option for pharmacological stress induction with a favorable profile specially in patients with COPD [5]. It is administered as a $0.4 \mathrm{mg}$ bolus injected over 15-20 s and followed by a 10-ml saline flush before the injection of the radiotracer. However, its clinical use has not been widely spread yet.

With ${ }^{15} \mathrm{O}-\mathrm{H}_{2} \mathrm{O}$ and ${ }^{82} \mathrm{Rb}$, the second phase can be performed without delay after the first study. However, with ${ }^{13} \mathrm{~N}$-ammonia the second study (stress or rest) is routinely delayed $30 \mathrm{~min}$ after the first study, to allow for radiotracer decay and the image acquisition is started a few seconds before the radiotracer injection. When a three-phase scan is performed, the CPT takes place after rest and before stress. CPT is evoked by submerging the patient's hand in ice water
$60 \mathrm{~s}$ before and $60 \mathrm{~s}$ after the beginning of the acquisition and administration of the tracer acquisition [6].

Furthermore, hybrid equipment offer the possibility to acquire a CT angiography (usually before the PET acquisition; in some centers, such CCTA is used for pre-selection of patients who can benefit from PET) and coronary artery calcium measurement within the same imaging session. Figure 2 depicts example protocols for PET and PET/CT.

Clinical protocols have been suggested for new promising compounds such as ${ }^{18} \mathrm{~F}$-flurpiridaz [7]. This fluorine18-based tracer extends the possibilities of perfusion imaging implementation through a regional rather than on-site cyclotron due to its longer half-life (110 min). Moreover, ${ }^{18} \mathrm{~F}$-flurpiridaz would facilitate exercise testing in contrast to other commonly used tracers [8].

\section{Dosimetry}

Several current standards for PET MPI, such as 3D acquisition and state-of-the-art software, offer the possibility for reduced radiation doses. A full ${ }^{82} \mathrm{Rb}$ 3D PET scan implies an exposure of $\approx 4.3 \mathrm{mSv}$, while for a single ${ }^{13} \mathrm{~N}$-ammonia $3 \mathrm{D}$ scan, the estimated the radiation dose is around 1 , and $0.8 \mathrm{mSv}$ for ${ }^{15} \mathrm{O}-\mathrm{H}_{2} \mathrm{O}$ [9]. The calculated exposure for ${ }^{18} \mathrm{~F}$-flurpiridaz has initially been estimated in $6.3 \mathrm{mSv}$ [7]. Notable, these doses are considerably lower than the ones for SPECT perfusion imaging.

The modality of hybrid PET/CT imaging poses an additional exposure due to the CCTA acquisition (1-18 $\mathrm{mSv}$ ) [10] within the same protocol. Nevertheless, with recent improvements in CT technology and the optimization of
Fig. 2 Acquisition protocols for PET-MPI. Example sequence for PET/CT MPI with ${ }^{13} \mathrm{~N}$-ammonia, ${ }^{15} \mathrm{O}-\mathrm{H}_{2} \mathrm{O}$ and ${ }^{82} \mathrm{Rb}$. CT-AC CT-based attenuation correction, $\mathrm{CaC}$ coronary calcium scan, CTA CT angiography
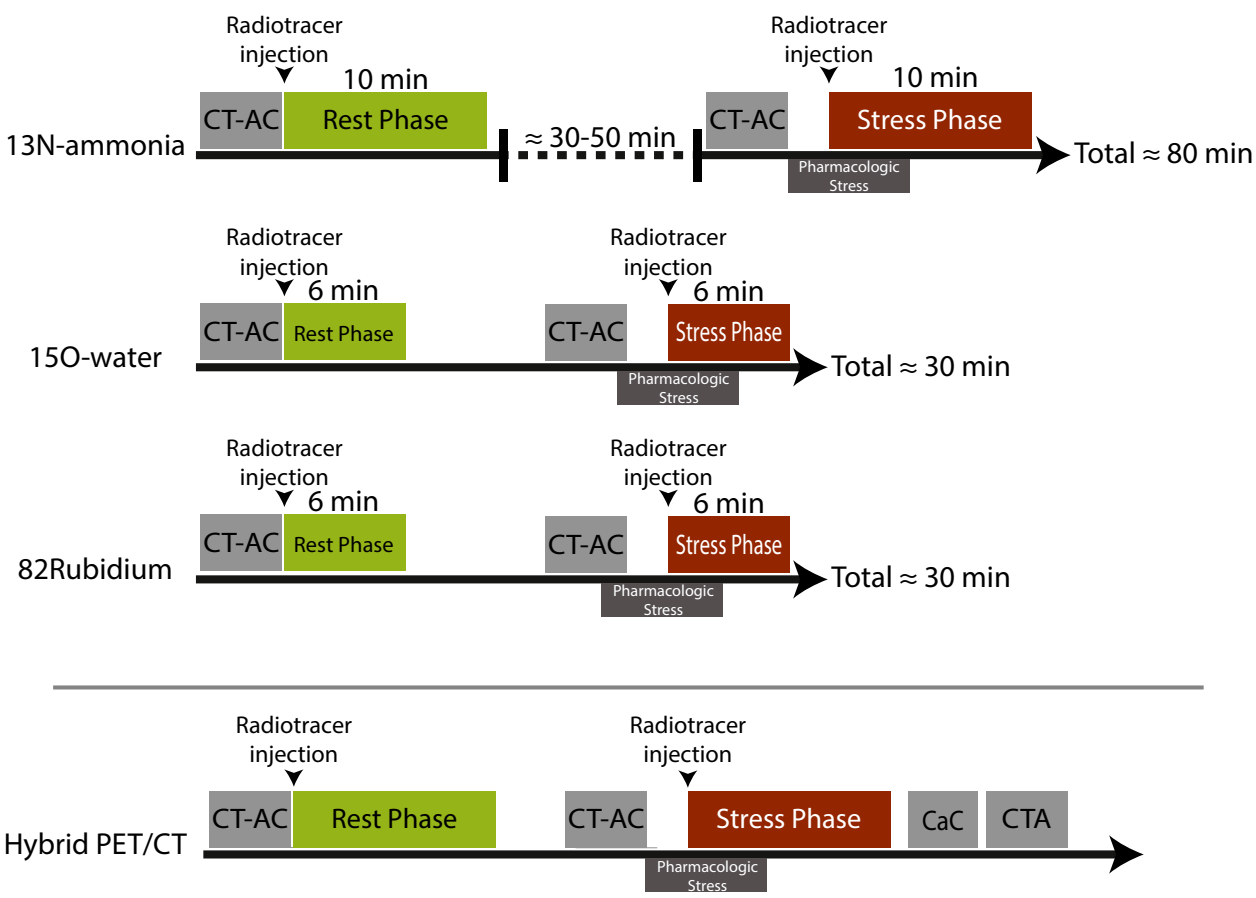
all-inclusive protocols with physiological tracers and prospectively triggered step-and-shoot CCTA, a full PET/CT imaging can currently be performed with a radiation dose under $10 \mathrm{mSv}$ [11].

\section{Perfusion radiotracers}

Several radiotracers have proved suitable for performing adequate and reproducible PET MPI. The most commonly used are Rubidium-82 ( $\left.{ }^{82} \mathrm{Rb}\right)$, Nitrogen-13 ammonia $\left({ }^{13} \mathrm{~N}\right.$-ammonia) and Oxygen-15 labeled water $\left({ }^{15} \mathrm{O}-\mathrm{H}_{2} \mathrm{O}\right)$, their general characteristics are summarized in Table 1 panel A.

${ }^{13} \mathrm{~N}$-ammonia This radiotracer represents an attractive compound with a relatively short half-life $(9.96 \mathrm{~min})$ and appealing uptake kinetics. ${ }^{13} \mathrm{~N}$-ammonia undergoes a celltrapping process due to its conversion to glutamine inside the cell. The favorable positron range $(2.53 \mathrm{~mm})$ and an $80 \%$ extraction fraction allow acquisition of intermediate to highquality images. The production of ${ }^{13} \mathrm{~N}$-ammonia requires an on-site cyclotron, limiting its availability. Nevertheless, smaller single-site cyclotrons presently offer unit dose production and use for ${ }^{13} \mathrm{~N}$-ammonia [4]. Research on results from this production is not yet available.

${ }^{15} \mathrm{O}-\mathrm{H}_{2} \mathrm{O} .{ }^{15} \mathrm{O}-\mathrm{H}_{2} \mathrm{O}$ portraits the ideal radiotracer for flow quantification due to its free diffusion through the cell membrane and proportional tissue uptake to flow. ${ }^{15} \mathrm{O}-\mathrm{H}_{2} \mathrm{O}$ has a physical half-life of $2.06 \mathrm{~min}$ and an intermediate positron range $(4.14 \mathrm{~mm})$. Remarkably, its extraction fraction is $100 \%$. Due to tracer characteristics, visual analysis of images is rather inadequate and absolute quantification or visualization of parametric images is preferred. Clinical research has indeed demonstrated the feasibility and diagnostic quality of parametric imaging with ${ }^{15} \mathrm{O}-\mathrm{H}_{2} \mathrm{O}$ [12]. This radiotracer also requires an on-site cyclotron for its production and unlike ${ }^{13} \mathrm{~N}$-ammonia, its half-life does not allow for nearby distribution.

${ }^{82}$ Rubidium. ${ }^{82} \mathrm{Rb}$ was one of the first introduced PET tracers. Notably, it does not require an on-site cyclotron for its production. Instead, ${ }^{82} \mathrm{Rb}$ can be obtained from commercially available elution generators which have a 5 -week shelf life, but availability has been hindered by limits in ${ }^{82}$ Strontium production. Presently, it constitutes the most used PET perfusion radiotracer in the USA. ${ }^{82} \mathrm{Rb}$ is potassium analog with a short physical half-life (76 s) with kinetic properties comparable to ${ }^{201}$ Thallium. It has a lower extraction fraction than other tracers as well as a longer positron range $(8.6 \mathrm{~mm})$ which limits the resolution of the generated images [4]. Nevertheless, due to its versatility, the greatest body of evidence and experience has been generated around ${ }^{82} \mathrm{Rb}$ perfusion studies.

Nowadays, research efforts have been dedicated to the development of new ${ }^{18}$ Fluorine-based perfusion radiotracers to attend to the growing need for providing access to PET perfusion radiotracers for a greater number of patients. ${ }^{18}$ Fluorine-based tracers offer a relatively long half-life of $110 \mathrm{~min}$. As such, compounds like ${ }^{18} \mathrm{~F}$-flurpiridaz, fluorodihydrorotenone $\left({ }^{18} \mathrm{~F}-\mathrm{FDHR}\right)[4]$ and several iterations of triphenyl phosphonium salts are currently under development.

${ }^{18} \mathrm{~F}$-Flurpiridaz is a structural analog of the insecticide pyridaben [13], an inhibitor of the NADH:ubiquinone oxidoreductase by competing for binding with ubiquinone

Table 1 PET perfusion radiotracer characteristics (panel A) and evidence-based status of implementation (panel B)

\begin{tabular}{|c|c|c|c|c|}
\hline & ${ }^{13} \mathrm{~N}$-ammonia & ${ }^{15} \mathrm{O}-\mathrm{H}_{2} \mathrm{O}$ & ${ }^{82}$ Rubidium & ${ }^{18} \mathrm{~F}$-flurpiridaz \\
\hline \multicolumn{5}{|l|}{ A } \\
\hline Half-life & $9.96 \mathrm{~min}$ & $2.06 \mathrm{~min}$ & $76 \mathrm{~s}$ & $109 \min$ \\
\hline Positron range (mm) & 2.53 & 4.14 & 8.6 & 1.03 \\
\hline $\begin{array}{l}\text { Myocardial extraction } \\
(\%)\end{array}$ & 80 & 100 & 65 & 94 \\
\hline Perfusion defect contrast & Intermediate & Intermediate & Lowest & Highest \\
\hline Cyclotron needed & Onsite or nearby & Onsite & No & In the region \\
\hline \multicolumn{5}{|l|}{ B } \\
\hline Validation & $\begin{array}{l}\text { Muzik et al. [55], Bol et al. } \\
\text { [56] }\end{array}$ & Bergmann et al. [57] & $\begin{array}{l}\text { Huang et al. [58], Lau- } \\
\text { tamäki et al. [59] }\end{array}$ & $\begin{array}{l}\text { Maddahi et al. [60], Berman } \\
\text { et al. [7], Packard et al. } \\
\text { [14] }\end{array}$ \\
\hline $\begin{array}{l}\text { Cross-comparison and } \\
\text { variability }\end{array}$ & $\begin{array}{l}\text { Nesterov et al. }(2012,), \\
\text { Nesterov et al } .(2017)\end{array}$ & $\begin{array}{l}\text { Nesterov et al. [61], Harms } \\
\text { et al. [62] }\end{array}$ & $\begin{array}{l}\text { Nesterov et al. [19], Kit- } \\
\text { kungvan et al. [63] }\end{array}$ & \\
\hline Cut-off exploration & Renaud et al. [64] & $\begin{array}{l}\text { Kajander et al. [50], Danad } \\
\text { et al. [35] }\end{array}$ & $\begin{array}{l}\text { Renaud et al. [64], Naya } \\
\text { et al. [34] }\end{array}$ & \\
\hline $\begin{array}{l}\text { Clinical testing for } \\
\text { outcomes }\end{array}$ & & $\begin{array}{l}\text { Neglia et al. [29], Danad } \\
\text { et al. [30] }\end{array}$ & & \\
\hline
\end{tabular}

References in italics are indexed abstracts 
without affecting the cardiac cell viability. It has demonstrated a short positron range $(1.03 \mathrm{~mm})$ and a high extraction fraction (94\%), offering improved image quality as documented by phase I and II trials [7]. Quantification over a wide range of MBF with ${ }^{18} \mathrm{~F}$-Flurpiridaz has been performed with engaging results that have demonstrated sensitive variations across CAD status [14].

${ }^{18}$ Fluorine-labeled phosphonium derivates $\left({ }^{18} \mathrm{~F}\right.$ - FATPs $)$ have demonstrated potential for PET MPI in preclinical studies. ${ }^{18} \mathrm{~F}$ - FATPs accumulate in cardiac myocytes due to their higher mitochondrial membrane potential and show intense myocardial uptake with sharp delineation of perfusion defects in animal models [15]. ${ }^{18} \mathrm{~F}$ - FATPs have been compared to ${ }^{13} \mathrm{~N}$-ammonia and favorable warrant further extension into clinical studies in the coming years [16].

\section{Dedicated software}

Several tailored softwares are available for automatic image reconstruction and blood flow quantification with minimum observer intervention such as QPS, syngoMBF, 4DM, PMOD, EcTB, CARIMAS, FlowQuant, HOQUTO, amongst others.

Due to heterogeneity in the models and reconstruction software application, recent research has been conducted to investigate whether estimations of flow with one or another model, software or scanner are to be considered interchangeable. There is evidence of good agreement between software packages for blood flow quantification with ${ }^{82} \mathrm{Rb}$ [17] and ${ }^{13} \mathrm{~N}$-ammonia [18] studies and it has been stated that reconstruction using a wide range of software, under the assumption of a 1-tissue compartment mode, has sufficient agreement to be used interchangeably. Nevertheless, it has also been demonstrated that agreement is not optimal between estimations derived from different kinetic models [19]. In summary, the choice of a kinetic model seems to constitute a greater influence in the feasibility of MBF comparison than the software used. Provided that the kinetic model applied will also depend on the used radiotracer, there is an evident added level of complexity in the matter.

Since current software alternatives allow quick and mostly automated estimations of parameters such as MBF, current challenges for software development and utilization mostly rely on the impending need for standardization, models and estimates validation in alternative populations and results optimization for attempting data pooling in a larger scale.

\section{Quantification of myocardial perfusion}

Dynamic acquisitions are used to map the distribution and concentration of the radiotracer during rest and stress throughout the myocardium by applying tracer kinetic models, which account for the influence of partial volume effect, spatial resolution, and motion. Usually, a one- or two-tissue compartment model is considered and first-pass activity distribution ( $K_{1}=$ extraction, which varies across tracers showing a roll-off effect at higher blood flows) are used to derive global and regional time-activity curves that yield estimation of the absolute myocardial blood flow. Features that increase the value of such estimations are the high temporal resolution of the technique, as well as the compulsory correction of photon attenuation. The magnitude of the underestimation of MBF at higher values couples with the necessary adjustment per tracer, but also increases the uncertainty around the equivalence of the quantification results. In this sense, ${ }^{82} \mathrm{Rb}$ conveys the lower extraction value, while that of ${ }^{13} \mathrm{~N}$-ammonia is perceivably better (see Perfusion radiotracers).

Furthermore, MBF estimations can be corrected to systematically account for the variability of the rate-pressure product (RPP) an index of myocardial oxygen consumption [20]. Since the relation between RPP and MBF is decoupled during stress, the correction is only made for rest MBF [21]. MBF is measured in milliliters per gram of tissue per minute $(\mathrm{ml} / \mathrm{g} / \mathrm{min})$; theoretically, this allows for comparable magnitudes across studies and pathologic conditions (see Dedicated software).

Stress myocardial blood flow and the ratio between stress and rest flow, named myocardial perfusion reserve (MPR), are used to evaluate the vasodilatory capacity of the coronary artery tree globally as well as regionally, based on several proposed segmentations such as the 17-segment model proposed by the American Heart Association or the vessel territory segmentation.

Furthermore, it is possible to compare MBF estimations obtained during the two or three scan phases. The ratio of CPT-MBF to rest MBF is known as the endothelial-dependent vasodilatory index (EDVI), while the ratio of stress $\mathrm{MBF}$ to rest MBF is known as the coronary flow reserve or myocardial perfusion reserve (MPR). Pharmacological or exercise stress evokes a compound vascular response and is suggested to represent the integrated coronary vascular function expressed by MPR.

Several factors exert a direct influence in the coronary vasodilator capacity and there is no simple relationship between pathologic-morphologic features (from non-invasive or invasive angiography) and perfusion defects. Notably, stress MBF and MPR have been recognized as important physiological parameters of cardiovascular health. These measurements have shown an inverse relationship with increasing severity of epicardial obstructive CAD and stress-induced regional perfusion defects [22]. Nevertheless, a marked variability is present across individual patients.

Since quantitative PET merges the perfusion dynamics of the main epicardial vessels and the microvascular circulatory 
detail in an integrative measurement, abnormal results in this evaluation should be carefully considered in accordance to factors that may hamper microvascular circulation additionally to flow-limiting subepicardial vessel stenoses. Hybrid imaging (PET/CT and PET/MR) has arisen in response to this need given the clinical importance of making this distinction.

Therefore, quantitative perfusion assessment may result useful to identify vasomotor abnormalities preceding overt CAD, improve diagnostic accuracy for CAD, estimate the burden of the disease in the full extent of the coronary system, potentially evaluate the effect of medical interventions, and enhance risk assessment for optimal prognostic evaluation.

\section{Diagnostic applications}

As for any technique, the pathologic horizon of applications of quantitative PET follows the natural history of the disease of interest (i.e. CAD). Evaluation of initial endothelial dysfunction in the microvascular detail and progression to non-obstructive and obstructive- or ischemic burden causing-CAD have been well described. The process of implementation for such purposes entails validation, agreement, threshold-establishing and clinical outcome studies. As such, available tracers demonstrate a differential implementation moment according to published reports. This is depicted in Table 1 panel B (however, references are not meant to be exhaustive). The understanding of this state of affairs is paramount for clinical investigators in order to advance efforts to achieve widespread clinical implementation.

\section{Microvascular disease and treatment monitoring}

Through the years, several reports using PET have demonstrated microvascular dysfunction in narrowly selected subsets of patients with specific cardiovascular risk factors such as smoking [23], dyslipidemia [24], hypertension [25], diabetes mellitus [26] and rheumatic diseases [27].

The EDVI and the MPR have been evaluated to detect impairment of coronary endothelial function and total vasodilatory function [6], respectively. Results from these studies suggest that non-invasive evaluation through quantitative PET can demonstrate impaired vasomotor function in the presence of cardiovascular risk factors. This may serve as a pathophysiological proxy of subclinical CAD, which might benefit from intense life-style modification.

Noteworthy, some studies have shown improvement in MBF dynamics through several interventions and after long-term risk factor modification [28]. This evidence suggests that treatment follow-up is feasible through quantitative PET. Nevertheless, doubts regarding the feasibility of this approach due to radiation exposure and costs should be further addressed. Notably, special patient populations may benefit from such an approach considering their morbidity profile (autoimmune disorders as vasculitis) and are to be contemplated in a patient-to-patient basis.

\section{Identifying and characterizing the burden of coronary artery disease}

PET has long been known to be an excellent study to detect regional myocardial perfusion defects. The clinical significance of the coronary lesions may be appropriately assessed with the advantage of sparing the patient from subsequent potentially unnecessary invasive procedures such as coronary angiography, which are not free of risk.

Currently, PET is considered the quantitative reference standard myocardial perfusion and it has demonstrated a good performance yielding a sensitivity of $81 \%$ and a specificity of $89 \%$ for angiographically significant coronary artery stenosis in the recently reported EVINCI trial [29]. Another major study, the PACIFIC trial, demonstrated directly that ${ }^{15} \mathrm{O}-\mathrm{H}_{2} \mathrm{O}$ PET conveys the best diagnostic performance (in comparison to SPECT and CCTA) with a sensitivity of $87 \%$ and a specificity of $84 \%$ for the detection of functionally significant lesions evaluated by fractional flow reserve (FFR) [30], the current gold standard for the functional assessment of coronary stenosis [31]. Figure 3 depicts a typical patient with intermediate risk of CAD investigated for myocardial ischemia.

Systematic evaluations of the diagnostic performance of PET have also been published. In one, Jaarsma et al. demonstrated an overall $84 \%$ sensitivity and $81 \%$ specificity across 15 studies in a per-patient basis considering a $\geq 50 \%$ luminal stenosis by invasive angiography as the reference standard [32]. Another report by Takx et al. documented a sensitivity and specificity of 84 and $87 \%$, respectively, and 83 and $89 \%$ at the vessel level. Although this study was based on 3 publications, it adequately utilized FFR as reference standard [31].

Flow quantification represents an additional feature that places PET above other non-invasive techniques in some aspects. One of the well-known applications is the scenario where perfusion is altered throughout several myocardial regions. In this scenario, traditional semi-quantitative assessment of PET imaging fails to comprehensively assess perfusion abnormalities due to its relative nature, considering the assumption that the region with the highest perfusion represents normality and is, therefore, used as reference.

Global reduction of perfusion can, on the other hand, be demonstrated through quantitative measurements, which are independent of a reference region. The determination of the etiology of the perfusion defect becomes a matter of additive diagnostic information since distinction form diffuse 


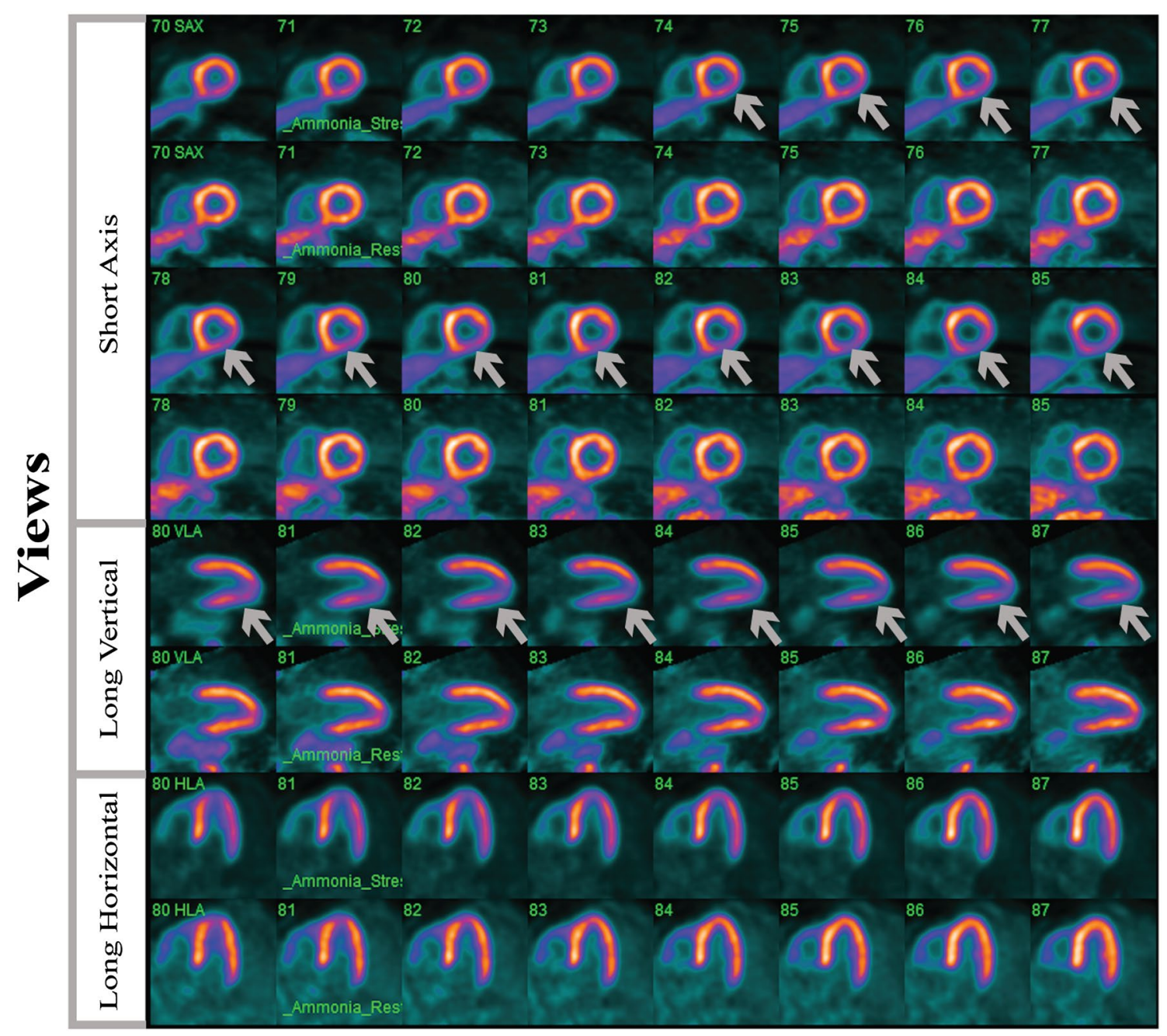

Fig. 3 PET MPI. PET MPI study of a 53 year-old patient undergoing evaluation for suspected CAD. The study shows moderate ischemia in the inferolateral wall (gray arrows), suggestive of left circumflex cor-

microvascular disease from multiple subepicardial vessel affection is difficult to address considering that also coronary abnormalities can play a role in hampering perfusion. As an example of the latter, reports of perfusion abnormalities are developing around coronary ectasia with and without atherosclerotic lesions [33]. The lack of such a distinction can lead to falsely negative results and underestimation of the cardiac risk in a given patient. In this clinical scenario, patients with trivascular involvement who present such a "pseudonormalization" phenomenon or "balanced" ischemia can be adequately studied with flow quantification (e.g., in Fig. 4), although this phenomenon may be less prevalent than originally considered.

More commonly, quantitative perfusion aids in the characterization of patients with single-, two- or three-vessel disease with a differential in perfusion impairment between coronary vessels. While, techniques like SPECT or even onary artery and/or right coronary artery lesion. PET positron emission tomography, $M P I$ myocardial perfusion imaging, $C A D$ coronary artery disease

semi-quantitative analysis with PET may report findings compatible with one-vessel CAD, quantification contributes with objective measures for the rest of the vessel territories providing a comprehensive view on the impact of CAD over the coronary circulation (e.g., in Fig. 5). Still, while a preserved MPR has shown a very high negative predictive value for excluding high-risk CAD, abnormalities in MPR alone cannot distinguish the level of the coronary tree affected by the suspected CAD [34].

Regional reductions in perfusion may, thus, be due to flow-limiting effects of coronary lesions or, as mentioned earlier, microvascular dysfunction related to cardiovascular risk factors, and the discrimination of effects cannot be addressed by the measurement alone even though several cutoff values have been studied to optimize obstructive CAD detection. For instance, the efforts conducted by Danad et al. have documented that $2.3 \mathrm{ml} / \mathrm{min} / \mathrm{g}$ for hyperemic $\mathrm{MBF}$ 
Fig. 4 MBF Quantification in multivessel CAD. PET

MPI study showing very

mild ischemic findings in the anteroapical and apicolateral segments in the semi-quantitative analysis. Nevertheless, quantitative assessment shows a reduced stress MBF and MPR in all arterial territories, achieving a better characterization of the trivascular disease, owing to a balanced ischemia or pseudonormalization phenomenon. $M B F$ myocardial blood flow, $C A D$ coronary artery disease, $P E T$ positron emission tomography, $M P I$ myocardial perfusion imaging, $C F R$ coronary flow reserve/myocardial perfusion reserve
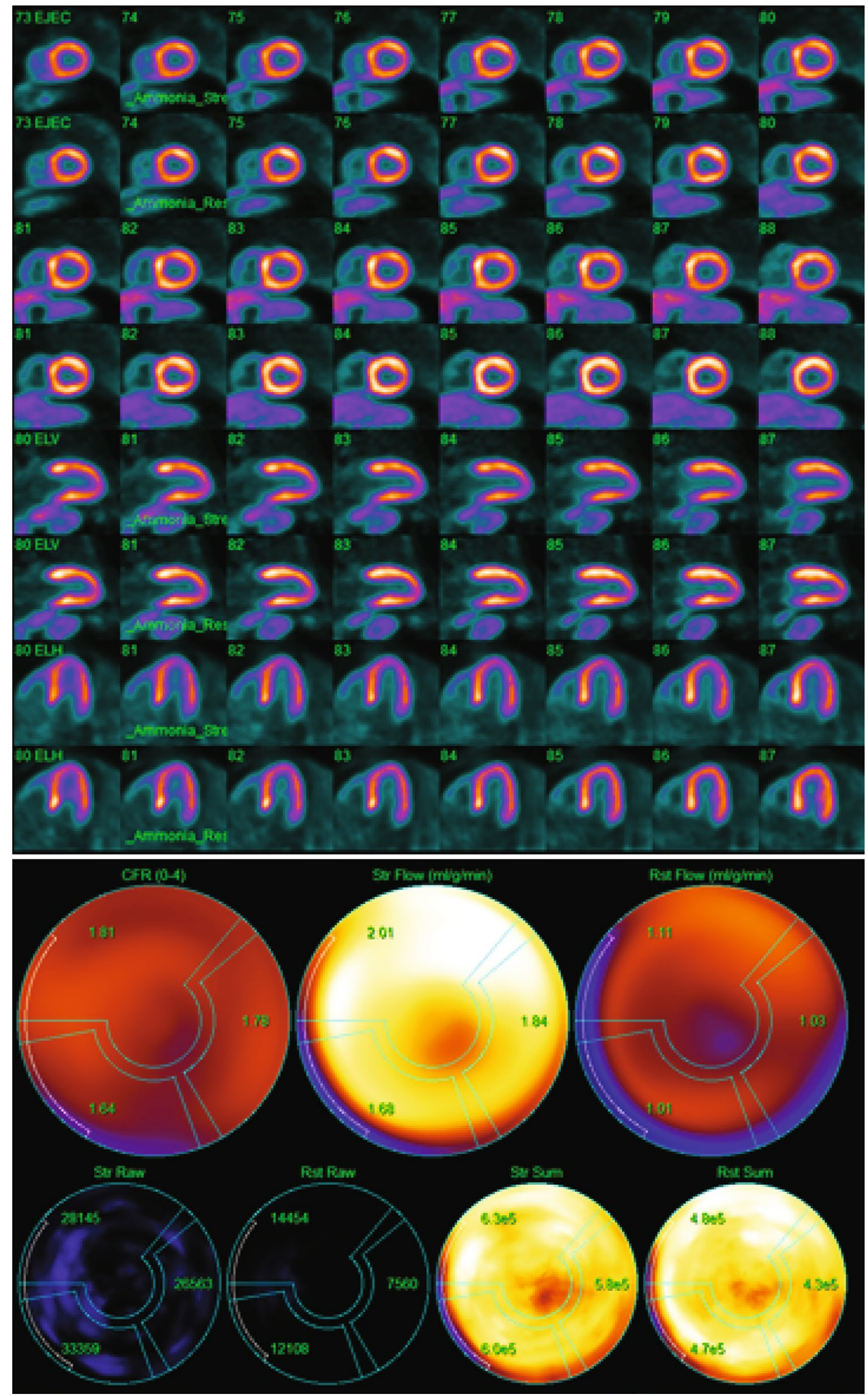

and 2.5 for MPR could represent optimal cutoff values for regional quantitative ${ }^{15} \mathrm{O}-\mathrm{H}_{2} \mathrm{O}$ PET [35] in the detection of invasive coronary angiography- and FFR-significant CAD [36].
Diagnostic performance improvement for $\mathrm{CAD}$ has been studied with similar results showing improvement in sensitivity and specificity by adding the quantitative evaluation, which in turn, does not require much more effort in 


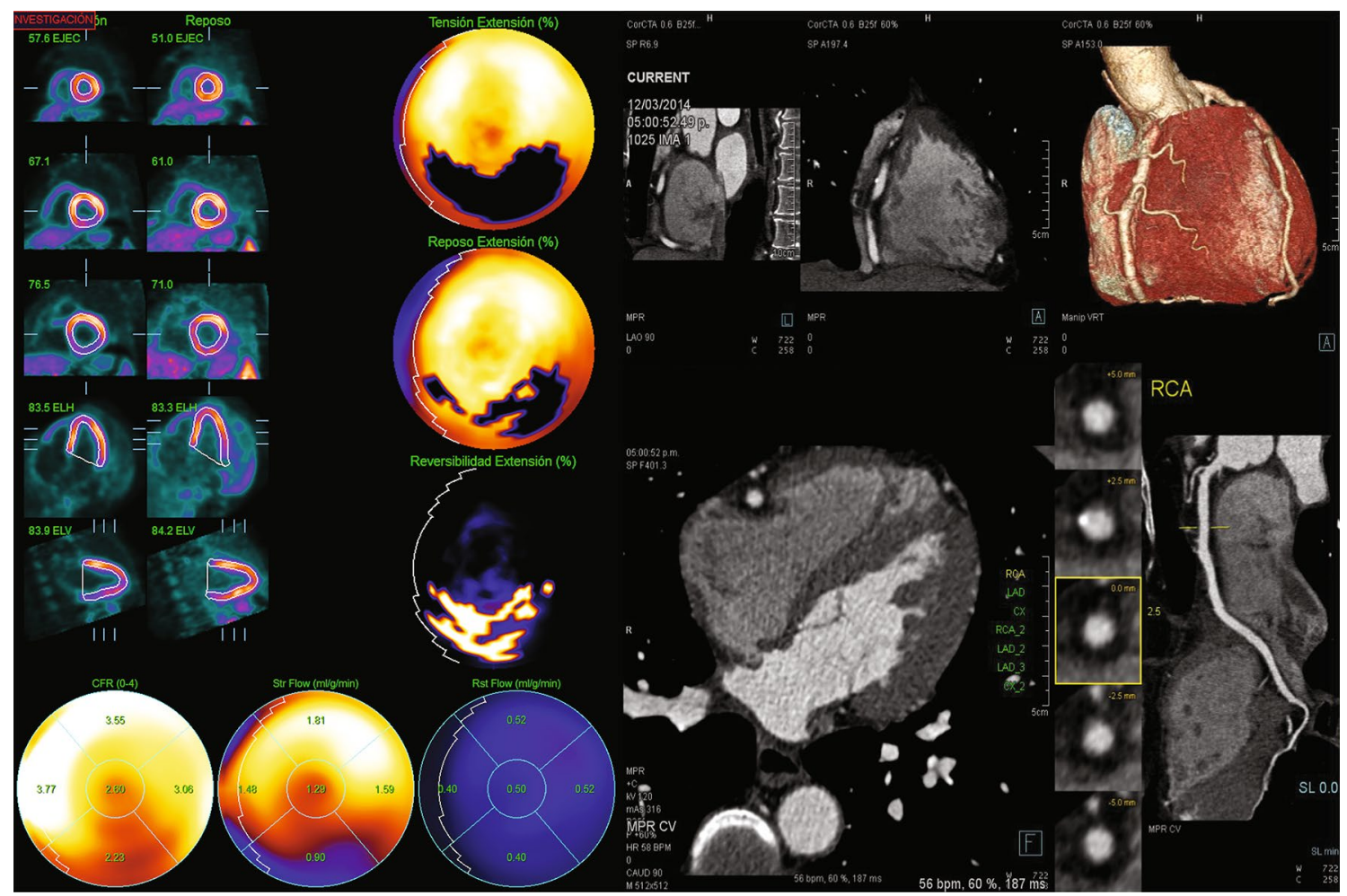

Fig. 5 Quantitative PET/CT (hybrid) in suspected CAD. PET MPI study showing severe semi-quantitative ischemia in the inferior ventricular wall, suggestive of RCA involvement. Nevertheless, quantitative assessment shows a reduced stress $\mathrm{MBF}(0.90 \mathrm{ml} / \mathrm{g} / \mathrm{min})$ and

the acquisition protocol making it an attractive technical addition (Fig. 3). Studies for establishing the most adequate threshold for abnormal stress MBF and MPR have been conducted and current evidence suggests that the former might yield a better diagnostic and prognostic value than the latter, in the setting of CAD detection [37].

Notably, there has also been information regarding the link between perfusion measurement and cardiac function. In this sense, it seems that the correlation between stress MBF and ventricular function and synchrony is better than that of MPR [38]. Further investigations should be encouraged to evaluate diagnostic and prognostic utility when parallel aspects of function are considered. This could be useful in patients in which only stress MBF protocols have been performed or prospectively, in patients in which stress-only should be performed due to radiation doses concerns.

It is evident then that MBF quantification and derived measures are to be considered within the integral PET MPI study as added values, which aid and improve the quality of the conclusions drawn from it. It has been stated that abnormal quantitative measurements are to be interpreted in addition to parallel anatomic and functional assessments to complement the information obtained and pinpoint the best way to proceed into the therapeutic process. It is here
MPR (2.23) in the same territory in comparison to the rest of the ventricular walls. CCTA documented non-calcified plaques with proximal RCA ectasia $(7 \mathrm{~mm})$

where hybrid modalities emerge and offer the added value of complementary anatomical and functional information. Whether its utilization will lead to improved patient outcomes is still to be answered. The essence of quantification as a "gatekeeper" for cardiac catheterization should strive for, in turn, moderating revascularization procedures.

In this way, the added value of flow quantification has expanded the possibilities of the technique from basic detection of advanced flow-limiting epicardial CAD to the broad evaluation of subclinical disease, the better characterization of its burden and the unmasking of multivessel perfusion affection.

It is relevant to circle back to the fact that different tracers currently exhibit different amount of evidence regarding their implementation, and that currently, although ${ }^{13} \mathrm{~N}$-ammonia and ${ }^{82} \mathrm{Rb}$ are used for clinical purposes, only data on the full diagnostic validation path for ${ }^{15} \mathrm{O}-\mathrm{H}_{2} \mathrm{O}$ is available. 


\section{Risk evaluation and prognostic performance}

Recently, clinical research has been trying to address another critical aspect of cardiac PET/CT application: whether its prognostic yield is relevant in comparison with more accessible techniques.

It has been shown that when applying the most common indications for PET MPI, which include chest pain, dyspnea or both, roughly half of the studies can be classified as normal according to the semi-quantitative visual analysis. Furthermore, with the possibility of measuring the MBF in absolute terms, recent studies have shown the correlation and incremental prognostic utility and added value of MPR measurements in the mid- and long-term follow-up, even after revascularization procedures [39]. Short-term follow-up studies have evaluated MPR as a relevant marker for prediction of cardiovascular events even within the subgroup of patients without regional perfusion abnormalities and previously infarcted patients. This supports the notion that MPR can be considered as an integrative marker for CV risk independent of previous $\mathrm{CV}$ events.

At the same time, the non-invasive measurement of MPR has been able to identify patients at increased risk of cardiac mortality in that a preserved MPR $(\geq 2.0)$ conveys an extremely low rate of cardiac mortality in contrast to patients with severely reduced MPR (hazard ratio of 5.6), even suggesting a "3-year warranty" event-free period. Moreover, MPR has shown utility in patient risk reclassification within the intermediate cardiovascular risk population (up to one third), which may constitute a major advantage for the technique considering that quantitative evaluation of $\mathrm{MBF}$ through PET MPI does not bear additional costs, procedures or radiation exposure. Additionally, PET MPI also results advantageous in the stress study of high-risk subsets of patients such as those with heart failure and obesity [40].

In the absence of epicardial stenosis, a decreased MPR is considered to document microvascular dysfunction caused by traditional $\mathrm{CV}$ risk factors, reflecting an early stage within the CAD horizon, while a preserved MPR portrays an excellent mid-term prognosis in patients with otherwise common risk factors. Moreover, quantitative PET MPI abnormalities have demonstrated to predict heart failure outcomes [41], and MPR has been evaluated as a stronger predictor for cardiac death than left ventricle ejection fraction [42].

Recently, this information has been systematically reviewed by our group-Juarez-Orozco and colleaguesto lay out and compare the reported prognostic significance of PET perfusion estimations [43]. We have concluded that perfusion estimations hold robust prognostic performance but only for the occurrence of major adverse $\mathrm{CV}$ events, while there is still a paucity of information regarding the occurrence of cardiac death alone. A directional comparison showed that MPR could be a better independent prognostic factor per se than stress MBF. Notably, this report underlines the substantial heterogeneity across studies, which represents a target for future development and standardization. Within the gathered studies, the report by Gupta et al. [44] assessed the differential significance and additive prognostic value of perfusion estimations (i.e., stress MBF and MPR). It seems then, that a concordantly hampered reduction in both estimations shows the highest association with cardiovascular mortality. Moreover, they reported that a preserved MPR can classify low-risk patients even when stress MBF is affected. This large report used mainly estimations provided by ${ }^{82} \mathrm{Rb}$ quantification and ${ }^{13} \mathrm{~N}$-ammonia in a minority of patients, a feature that raises the concern of the error introduced by conceding the exchangeability of estimations from different tracers.

Importantly, their results should be considered within the context of global perfusion measurements because in contrast, the evaluated study by Maaniitty et al. [45] has documented the prognostic value of regional stress MBF in the sequential application of coronary computed tomography angiography (CCTA) and ${ }^{15} \mathrm{O}-\mathrm{H}_{2} \mathrm{O}$ PET supporting its robustness and applicability.

An additional concern raise by our systematic investigation is that the prognostic value of a technique, particularly in cardiovascular medicine, must be understood from the operationalization of outcomes of interest. As suspected, profound differences were found in the amount and type of events recorded as MACEs, which contributed to hamper the potential pooling of reported results.

Finally, a recent report by Smulders et al. addressed the prognostic value of a negative result in non-invasive imaging with several techniques (including PET) concluding that overall, such a result conveys excellent prognosis in known or suspected CAD [46].

Findings from this type of studies may be valuable in determining which patients could be adequately selected for medical treatment alone and which would need further testing or interventional procedures to improve mortality rates. Moreover, it is likely that quantitative perfusion evaluation through PET could aid in treatment monitoring (for both primary and secondary prevention) since the intrinsic prognostic value of MBF and MFR is robustly documented. For instance, Schindler et al. documented the improvement in vascular reactivity as measured by quantitative perfusion in diabetic patients subjected to glucoselowering treatment [47]. Also, Quercoli et al. have demonstrated an improvement in coronary vasomotor function (both during CPT and pharmacological stress) after gastric-bypass in morbidly obese subjects [48]. However, 
whether vasomotor function can constitute a robust target for risk modification remains to be tested.

Furthermore, recent studies are unequivocally shifting towards the inclusion of enriched and geographically diverse groups of patients in an attempt to enhance the generalizable implications of its results.

\section{Hybrid modalities}

Nowadays, most of the available centers for PET scanning utilize it in the form of hybrid equipment, mostly in combination with CT scan (PET/CT), and in a much more discreet, but growing degree, with MRI (PET/MRI).

This is important because PET/CT provides complementary information. On one side, it documents the evaluation of intrinsic physiological and functional processes through PET (depending on the selected radiotracer and quantitative analysis) and on the other side, it delivers an accurate anatomical evaluation of the heart, the coronary vessels and related structures. This allows us to frame a comprehensive image of the disease, compensating for each technique's individual drawbacks at the same time [49].

The addition of CCTA and coronary calcium scoring helps to clarify the etiology of the perfusion defects, which could be attributed to flow-limiting lesions, unexpected artifacts, or false positive results (inherent to every technique). This is due to the ability of contrastenhanced CCTA to directly characterize coronary stenosis and plaque gross structure. CCTA has established itself as a useful method in the clinical scenario for resolving unclear PET MPI results, defining multivessel disease in "at glance" normal perfusion studies and excluding significant $\mathrm{CAD}$ in low and intermediate cardiovascular risk patients [50].

Several studies have shown improved sensitivity, specificity and accuracy of hybrid PET/CT over each study alone for the diagnosis of clinically significant CAD [37]. Furthermore, the information provided can support or discourage unnecessary invasive angiographic assessments and has demonstrated value in risk stratification and prognosis [51]. Although a diagnostic approach in which CCTA is performed to exclude significant CAD and spare MPI has proven to be cost effective and to avoid an increase in utilization of other imaging methods [52], the increasingly evolving notion of benefit from complementary information from hybrid methods is evident as exemplified in Fig. 5.

The theoretical advantages of such technical features of hybrid modalities converge in the improved evaluation of subclinical and overt CAD and in the determination of early pathophysiological changes of the disease (intermediate risk) as well as complex disease in patients with long-standing $\mathrm{CV}$ disease features (multivessel disease with complex anatomy or chronic occlusions). However, this is stance is now without controversy. In fact recently, the results from the PACIFIC trial showed that hybrid modalities' performance was not superior to that of individual techniques in the detection of functionally significant CAD [30] (besides demonstrating that PET conveys the best diagnostic performance overall). As such, open questions remain concerning hybrid technique application and whether the term itself represents the simultaneous use of individual techniques, the utility of concordant results for individual techniques or the sequential interpretation when two techniques are performed. In this last regard, a recent study by Maaniitty et al. [45] has documented the prognostic utility of a selective sequential application of ${ }^{15} \mathrm{O}-\mathrm{H}_{2} \mathrm{O}$ PET when evaluating dubious or positive $\mathrm{CT}$ angiography results. Their results have shown that the mortality rate for patients with CT-obstructive CAD but without ischemia is comparable to that of patients in whom obstructive CAD has been excluded.

Nowadays, PET/MR scanners offer attractive possibilities for combining measures of ventricular function, perfusion, viability, and infarct scar for evaluation of ischemic heart disease due to the intrinsic advantages of MR (no radiation exposure and higher spatial resolution). Then again, complementary value of PET/MR has to be demonstrated above the overlapping nature of the information provided. It has been stated that the greater contribution of PET/MR in the realm of cardiovascular disease will crystallize beyond CAD evaluation, for example, in the characterization of cardiomyopathies or infiltrative diseases [53].

In the clinical scenario, hybrid imaging could be justified according to the clinical question for the individual patients and the need for physiological data and precise anatomic delineation must be considered a feasible advantage of the equipment more than an automatic choice linked to the possibility of obtaining additional information [54].

\section{Conclusion}

PET myocardial perfusion currently represents the reference technique for quantitative perfusion assessment. The contribution of MBF and MPR beyond traditional semiquantitative evaluation of perfusion abnormalities improves diagnostic and prognostic performance in patients with CAD. Quantification of MBF with PET allows detection of early vasomotor abnormalities linked to subclinical disease; it permits treatment evaluation and it offers accurate diagnostic evaluation of patients with known or suspected atherosclerosis. 
Additionally, its prognostic value is steadily being recognized. New developments in available tracers and hybrid modalities will enhance the possibilities of accurate noninvasive imaging in several clinical scenarios. Therefore, the clinical value of PET quantitative perfusion is expected to improve patient outcomes and optimize therapeutic decisions, which constitute key elements for the future of cardiovascular medicine.

Author Contribution's LEJO-Literature Search, Review, Writing, Editing, Content Planning, JRCM-Literature Search, Review, Writing, GYGN—Literature Search, Review, Writing, LWL-Literature Search, Review, Writing, Editing, LJCE-Literature Search, Review, Writing, Editing, JK-Literature Search, Review, Writing, Editing, Content Planning, EA-Literature Search, Review, Writing, Editing, Content Planning.

\section{Compliance with ethical standards}

Conflict of interest Juarez-Orozco, Cruz-Mendoza, Guinto-Nishimura, Walls-Laguarda, Casares-Echeverría, Meave-Gonzalez, Knuuti and Alexanderson declare that they have no conflict of interest.

Open Access This article is distributed under the terms of the Creative Commons Attribution 4.0 International License (http://creativeco mmons.org/licenses/by/4.0/), which permits unrestricted use, distribution, and reproduction in any medium, provided you give appropriate credit to the original author(s) and the source, provide a link to the Creative Commons license, and indicate if changes were made.

\section{References}

1. Nakazato R, Berman DS, Alexanderson E, Slomka P (2013) Myocardial perfusion imaging with PET. Imaging Med 5:35-46. https ://doi.org/10.2217/iim.13.1

2. Bateman TM, Heller GV, McGhie AI et al (2006) Diagnostic accuracy of rest/stress ECG-gated Rb-82 myocardial perfusion PET: comparison with ECG-gated Tc-99m sestamibi SPECT. J Nucl Cardiol 13:24-33. https://doi.org/10.1016/j.nuclcard.2005.12.004

3. Nakazato R, Berman DS, Dey D et al (2012) Automated quantitative $\mathrm{Rb}-82$ 3D PET/CT myocardial perfusion imaging: normal limits and correlation with invasive coronary angiography. J Nucl Cardiol 19:265-276. https://doi.org/10.1007/s12350-011-9496-3

4. Maddahi J, Packard RRS (2014) Cardiac PET perfusion tracers: current status and future directions. Semin Nucl Med 44:333-343. https://doi.org/10.1053/j.semnuclmed.2014.06.011

5. Golzar Y, Doukky R (2014) Regadenoson use in patients with chronic obstructive pulmonary disease: the state of current knowledge. Int J Chronic Obstr Pulm Dis 9:129-137. https://doi. org/10.2147/COPD.S56879

6. Schindler TH, Nitzsche EU, Olschewski M et al (2004) PET-measured responses of MBF to cold pressor testing correlate with indices of coronary vasomotion on quantitative coronary angiography. J Nucl Med 45:419-428

7. Berman DS, Maddahi J, Tamarappoo BK et al (2013) Phase II safety and clinical comparison with single-photon emission computed tomography myocardial perfusion imaging for detection of coronary artery disease: flurpiridaz $\mathrm{F} 18$ positron emission tomography. J Am Coll Cardiol 61:469-477. https://doi. org/10.1016/j.jacc.2012.11.022

8. Iskandrian AE, Dilsizian V, Garcia EV et al (2017) Myocardial perfusion imaging: lessons learned and work to be done-update. J Nucl Cardiol. https://doi.org/10.1007/s12350-017-1093-7

9. Knuuti J (2009) Integrated positron emission tomography/computed tomography (PET/CT) in coronary disease. Heart 95:14571463. https://doi.org/10.1136/hrt.2008.151944

10. Mangla A, Oliveros E, Williams KA, Kalra DK (2017) Cardiac imaging in the diagnosis of coronary artery disease. Curr Probl Cardiol 42:316-366. https://doi.org/10.1016/j.cpcar diol.2017.04.005

11. Kajander S, Ukkonen H, Sipilä H et al (2009) Low radiation dose imaging of myocardial perfusion and coronary angiography with a hybrid PET/CT scanner. Clin Physiol Funct Imaging 29:81-88. https://doi.org/10.1111/j.1475-097X.2008.00838.X

12. Harms HJ, Knaapen P, de Haan S et al (2011) Automatic generation of absolute myocardial blood flow images using [15O]H2O and a clinical PET/CT scanner. Eur J Nucl Med Mol Imaging 38:930-939. https://doi.org/10.1007/s00259-011-1730-3

13. Yalamanchili P, Wexler E, Hayes M et al (2007) Mechanism of uptake and retention of F-18 BMS-747158-02 in cardiomyocytes: a novel PET myocardial imaging agent. J Nucl Cardiol 14:782-788. https://doi.org/10.1016/j.nuclcard.2007.07.009

14. Packard RRS, Huang S-C, Dahlbom M et al (2014) Absolute quantitation of myocardial blood flow in human subjects with or without myocardial ischemia using dynamic flurpiridaz $\mathrm{F} 18$ PET. J Nucl Med 55:1438-1444. https://doi.org/10.2967/jnume d.114.141093

15. Higuchi T, Fukushima K, Rischpler C et al (2011) Stable delineation of the ischemic area by the PET perfusion tracer 18F-fluorobenzyl triphenyl phosphonium after transient coronary occlusion. J Nucl Med 52:965-969. https://doi. org/10.2967/jnumed.110.085993

16. Kim D-Y, Kim HS, Reder S et al (2015) Comparison of $18 \mathrm{~F}$-labeled fluoroalkylphosphonium cations with $13 \mathrm{~N}-\mathrm{NH} 3$ for PET myocardial perfusion imaging. J Nucl Med 56:1581-1586. https://doi.org/10.2967/jnumed.115.156794

17. Dekemp RA, Declerck J, Klein R et al (2013) Multisoftware reproducibility study of stress and rest myocardial blood flow assessed with 3D dynamic PET/CT and a 1-tissue-compartment model of 82Rb kinetics. J Nucl Med 54:571-577. https://doi. org/10.2967/jnumed.112.112219

18. Slomka PJ, Alexanderson E, Jácome R et al (2012) Comparison of clinical tools for measurements of regional stress and rest myocardial blood flow assessed with $13 \mathrm{~N}$-ammonia PET/ CT. J Nucl Med 53:171-181. https://doi.org/10.2967/jnume d.111.095398

19. Nesterov SV, Deshayes E, Sciagrà R et al (2014) Quantification of myocardial blood flow in absolute terms using (82)Rb PET imaging: the RUBY-10 study. JACC Cardiovasc Imaging 7:1119-1127. https://doi.org/10.1016/j.jcmg.2014.08.003

20. Czernin J, Müller P, Chan S et al (1993) Influence of age and hemodynamics on myocardial blood flow and flow reserve. Circulation 88:62-69. https://doi.org/10.1161/01.CIR.88.1.62

21. Uren NG, Melin JA, De Bruyne B et al (1994) Relation between myocardial blood flow and the severity of coronary-artery stenosis. N Engl J Med 330:1782-1788. https://doi.org/10.1056/ NEJM199406233302503

22. Di Carli M, Czernin J, Hoh CK et al (1995) Relation among stenosis severity, myocardial blood flow, and flow reserve in patients with coronary artery disease. Circulation 91:1944-1951. https:// doi.org/10.1161/01.CIR.91.7.1944

23. Dayanikli F, Grambow D, Muzik O et al (1994) Early detection of abnormal coronary flow reserve in asymptomatic men at high risk 
for coronary artery disease using positron emission tomography. Circulation 90:808-817. https://doi.org/10.1161/01.CIR.90.2.808

24. Kaufmann PA, Gnecchi-Ruscone T, Schäfers KP et al (2000) Low density lipoprotein cholesterol and coronary microvascular dysfunction in hypercholesterolemia. J Am Coll Cardiol 36:103-109

25. Alexanderson E, Jacome R, Jimenez-Santos M et al (2012) Evaluation of the endothelial function in hypertensive patients with 13N-ammonia PET. J Nucl Cardiol 19:979-986. https://doi. org/10.1007/s12350-012-9584-z

26. Alexanderson E, Rodriguez-Valero M, Martinez A et al (2009) Endothelial dysfunction in recently diagnosed type 2 diabetic patients evaluated by PET. Mol Imaging Biol 11:1-5. https://doi. org/10.1007/s11307-008-0173-0

27. Alexánderson E, Ochoa JM, Calleja R et al (2010) Endothelial dysfunction in systemic lupus erythematosus: evaluation with 13N-ammonia PET. J Nucl Med 51:1927-1931. https://doi. org/10.2967/jnumed.110.078212

28. Gould KL, Ornish D, Scherwitz L et al (1995) Changes in myocardial perfusion abnormalities by positron emission tomography after long-term, intense risk factor modification. JAMA 274:894901. https://doi.org/10.1001/jama.274.11.894

29. Neglia D, Rovai D, Caselli C et al (2015) Detection of significant coronary artery disease by noninvasive anatomical and functional imaging. Circ Cardiovasc Imaging 8:e002179-e002179. https:// doi.org/10.1161/CIRCIMAGING.114.002179

30. Danad I, Raijmakers PG, Driessen RS et al (2017) Comparison of coronary CT angiography, SPECT, PET, and hybrid imaging for diagnosis of ischemic heart disease determined by fractional flow reserve. JAMA Cardiol 2:1100-1107. https://doi.org/10.1001/ jamacardio.2017.2471

31. Takx RAP, Blomberg BA, El Aidi H et al (2015) Diagnostic accuracy of stress myocardial perfusion imaging compared to invasive coronary angiography with fractional flow reserve meta-analysis. Circ Cardiovasc Imaging 8:e02666-e002666. https://doi. org/10.1161/circimaging.114.002666

32. Jaarsma C, Leiner T, Bekkers SC et al (2012) Diagnostic performance of noninvasive myocardial perfusion imaging using single-photon emission computed tomography, cardiac magnetic resonance, and positron emission tomography imaging for the detection of obstructive coronary artery disease: a meta-anal. J Am Coll Cardiol 59:1719-1728. https://doi.org/10.1016/j. jacc.2011.12.040

33. Ziadi MC, Dekemp RA, Williams K et al (2012) Does quantification of myocardial flow reserve using rubidium-82 positron emission tomography facilitate detection of multivessel coronary artery disease? J Nucl Cardiol 19:670-680. https://doi. org/10.1007/s12350-011-9506-5

34. Naya M, Murthy V, Taqueti V et al (2014) Preserved coronary flow reserve effectively excludes high- risk coronary artery disease on angiography. J Nucl Med 55:248-255. https://doi. org/10.2967/jnumed.113.121442.Preserved

35. Danad I, Uusitalo V, Kero T et al (2014) Quantitative assessment of myocardial perfusion in the detection of significant coronary artery disease: cutoff values and diagnostic accuracy of quantitative [(15)O]H2O PET imaging. J Am Coll Cardiol 64:1464-1475. https://doi.org/10.1016/j.jacc.2014.05.069

36. Gould KL, Johnson NP, Bateman TM et al (2013) Anatomic versus physiologic assessment of coronary artery disease. Role of coronary flow reserve, fractional flow reserve, and positron emission tomography imaging in revascularization decision-making. J Am Coll Cardiol 62:1639-1653. https://doi.org/10.1016/j. jacc.2013.07.076

37. Danad I, Raijmakers PG, Appelman YE et al (2013) Hybrid imaging using quantitative $\mathrm{H} 215 \mathrm{O}$ PET and CT-based coronary angiography for the detection of coronary artery disease. J Nucl Med. https://doi.org/10.2967/jnumed.112.104687
38. Juárez-Orozco LELE, Alexanderson E, Dierckx RARA et al (2016) Stress myocardial blood flow correlates with ventricular function and synchrony better than myocardial perfusion reserve: a nitrogen-13 ammonia PET study. J Nucl Cardiol. https://doi. org/10.1007/s12350-016-0669-y

39. Herzog BA, Husmann L, Valenta I et al (2009) Long-term prognostic value of $13 \mathrm{~N}$-ammonia myocardial perfusion positron emission tomography added value of coronary flow reserve. J Am Coll Cardiol 54:150-156. https://doi.org/10.1016/j. jacc.2009.02.069

40. Chow BJW, Dorbala S, Di Carli MF et al (2014) Prognostic value of PET myocardial perfusion imaging in obese patients. JACC Cardiovasc Imaging 7:278-287. https://doi.org/10.1016/j. jcmg.2013.12.008

41. Majmudar MD, Murthy VL, Shah RV et al (2015) Quantification of coronary flow reserve in patients with ischaemic and non-ischaemic cardiomyopathy and its association with clinical outcomes. Eur Heart J Cardiovasc Imaging 16:900-909. https:// doi.org/10.1093/ehjci/jev012

42. Tio RA, Dabeshlim A, Siebelink H-MJ et al (2009) Comparison between the prognostic value of left ventricular function and myocardial perfusion reserve in patients with ischemic heart disease. $\mathrm{J}$ Nucl Med 50:214-219. https://doi.org/10.2967/jnumed.108.05439 5

43. Juárez-Orozco LE, Tio RA, Alexanderson E et al (2017) Quantitative myocardial perfusion evaluation with positron emission tomography and the risk of cardiovascular events in patients with coronary artery disease: a systematic review of prognostic studies. Eur Heart J Cardiovasc Imaging. https://doi.org/10.1093/ehjci /jex331

44. Gupta A, Taqueti VR, van de Hoef TP et al (2017) Integrated noninvasive physiological assessment of coronary circulatory function and impact on cardiovascular mortality in patients with stable coronary artery disease. Circulation. https://doi.org/10.1161/ CIRCULATIONAHA.117.029992

45. Maaniitty T, Stenström I, Bax JJ et al (2017) Prognostic value of coronary $\mathrm{CT}$ angiography with selective PET perfusion imaging in coronary artery disease. JACC Cardiovasc Imaging. https://doi. org/10.1016/j.jcmg.2016.10.025

46. Smulders MW, Jaarsma C, Nelemans PJ et al (2017) Comparison of the prognostic value of negative non-invasive cardiac investigations in patients with suspected or known coronary artery diseasea meta-analysis. Eur Hear J-Cardiovasc Imaging 18:980-987. https://doi.org/10.1093/ehjci/jex014

47. Schindler TH, Cadenas J, Facta AD et al (2009) Improvement in coronary endothelial function is independently associated with a slowed progression of coronary artery calcification in type 2 diabetes mellitus. Eur Heart J 30:3064-3073. https://doi.org/10.1093/ eurheartj/ehp482

48. Quercioli A, Montecucco F, Pataky Z et al (2013) Improvement in coronary circulatory function in morbidly obese individuals after gastric bypass-induced weight loss: relation to alterations in endocannabinoids and adipocytokines. Eur Heart J 34:2063-2073. https://doi.org/10.1093/eurheartj/eht085

49. Saraste A, Knuuti J (2012) Cardiac PET, CT, and MR: what are the advantages of hybrid imaging? Curr Cardiol Rep 14:24-31. https://doi.org/10.1007/s11886-011-0231-0

50. Kajander S, Joutsiniemi E, Saraste M et al (2010) Cardiac positron emission tomography/computed tomography imaging accurately detects anatomically and functionally significant coronary artery disease. Circulation 122:603-613. https://doi.org/10.1161/CIRCU LATIONAHA.109.915009

51. Naya M, Murthy VL, Foster CR et al (2013) Prognostic interplay of coronary artery calcification and underlying vascular dysfunction in patients with suspected coronary artery disease. J Am Coll Cardiol 61:2098-2106. https://doi.org/10.1016/j.jacc.2013.02.029 
52. Fiechter M, Ghadri JR, Wolfrum M et al (2012) Downstream resource utilization following hybrid cardiac imaging with an integrated cadmium-zinc-telluride/64-slice CT device. Eur J Nucl Med Mol Imaging 39:430-436. https://doi.org/10.1007/s0025 9-011-1999-2

53. Rischpler C, Nekolla SG, Dregely I, Schwaiger M (2013) Hybrid PET/MR imaging of the heart: potential, initial experiences, and future prospects. J Nucl Med 54:402-415. https://doi.org/10.2967/ jnumed.112.105353

54. Truong QA, Gewirtz H (2014) Cardiac PET-CT for monitoring medical and interventional therapy in patients with CAD: PET alone versus hybrid PET-CT? Curr Cardiol Rep 16:460. https:// doi.org/10.1007/s11886-013-0460-5

55. Muzik O, Beanlands RS, Hutchins GD et al (1993) Validation of nitrogen-13-ammonia tracer kinetic model for quantification of myocardial blood flow using PET. J Nucl Med 34:83-91

56. Bol A, Melin JA, Vanoverschelde JL et al (1993) Direct comparison of [13 N]ammonia and [15O]water estimates of perfusion with quantification of regional myocardial blood flow by microspheres. Circulation 87:512-525. https://doi.org/10.1161/01. cir.87.2.512

57. Bergmann SR, Herrero P, Markham J et al (1989) Noninvasive quantitation of myocardial blood flow in human subjects with oxygen-15-labeled water and positron emission tomography. J Am Coll Cardiol 14:639-652. https://doi.org/10.1016/07351097(89)90105-8

58. Huang SC, Williams BA, Krivokapich J et al (1989) Rabbit myocardial 82Rb kinetics and a compartmental model for blood flow estimation. Am J Physiol 256:H1156-H1164. https://doi. org/10.1152/ajpheart.1989.256.4.H1156
59. Lautamäki R, George RT, Kitagawa K et al (2009) Rubidium-82 PET-CT for quantitative assessment of myocardial blood flow: validation in a canine model of coronary artery stenosis. Eur J Nucl Med Mol Imaging 36:576-586. https://doi.org/10.1007/ s00259-008-0972-1

60. Maddahi J, Czernin J, Lazewatsky J et al (2011) Phase I, firstin-human study of BMS747158, a novel 18F-labeled tracer for myocardial perfusion PET: dosimetry, biodistribution, safety, and imaging characteristics after a single injection at rest. J Nucl Med 52:1490-1498. https://doi.org/10.2967/jnumed.111.092528

61. Nesterov SV, Han C, Mäki M et al (2009) Myocardial perfusion quantitation with 15O-labelled water PET: high reproducibility of the new cardiac analysis software (Carimas). Eur J Nucl Med Mol Imaging 36:1594-1602. https://doi.org/10.1007/s0025 9-009-1143-8

62. Harms HJ, Nesterov SV, Han C et al (2014) Comparison of clinical non-commercial tools for automated quantification of myocardial blood flow using oxygen-15-labelled water PET/CT. Eur Heart J Cardiovasc Imaging 15:431-441. https://doi.org/10.1093/ ehjci/jet177

63. Kitkungvan D, Johnson NP, Roby AE et al (2017) Routine clinical quantitative rest stress myocardial perfusion for managing coronary artery disease: clinical relevance of test-retest variability. JACC Cardiovasc Imaging 10:565-577. https://doi.org/10.1016/j. jemg.2016.09.019

64. Renaud JM, Dasilva JN, Beanlands RSB, Dekemp RA (2013) Characterizing the normal range of myocardial blood flow with 82rubidium and $13 \mathrm{~N}$-ammonia PET imaging. J Nucl Cardiol 20:578-591. https://doi.org/10.1007/s12350-013-9721-3 